\title{
Elternrecht, staatlicher Schulerziehungsauftrag und Entfaltungsfreiheit des Kindes
}

\section{Vorbemerkung}

Der Streit um das Verhältnis des Erziehungsrechts der Eltern zum Schulerziehungsrecht des Staates ist so alt wie die öffentliche Schule selbst. Dabei beanspruchen beide Seiten für sich höchst ambivalente Vorrechte, wobei nur in einem Punkt Einigkeit besteht - der Unterordnung des (grundrechts-) )unmündigen Kindes unter den Kompetenzstreit zwischen Elternhaus und Schule. Nach dem Grundgesetz stehen sich in Art. 6 Abs. 2 GG das Elternrecht und in Art. 7 Abs. I GG das aus der Schulaufsicht des Staates begründete staatliche Schulerziehungsrecht gegenüber, denen das Recht des Kindes auf freie Entfaltung der Persönlichkeit gem. Art. 2 Abs. I i. V. m. Art. x Abs. I GG entgegensteht. Da klare verfassungsrechtliche Vorgaben über die Abgrenzung der einzelnen Grundrechte fehlen, hat sich der Streit hierüber weitgehend vor die Gerichte verlagert. Es soll Aufgabe dieser Arbeit sein, anhand einer historischen Analyse darzulegen, welchen objektiven gesellschaftlichen Bedingungen die Kompetenzzuweisung von Erziehungsrechten entspricht, was dies für die Persönlichkeit des Kindes bedeutet und welche Möglichkeiten einer Neubestimmung im Interesse des Kindes bestehen.

\section{Zur historischen Entwicklung}

Mit der Säkularisierung des Schulwesens im 16./17. Jahrhundert entsteht die Frage nach dem Verhältnis des Erziehungsrechts der Eltern zum Schulerziehungsrecht des Staates. Indem der Staat zunächst durch die Errichtung sog. Fürsten- und Landesschulen in Konkurrenz zu kirchlichen Schulen tritt, versucht er, nicht nur den Einfluß der Kirche aus der Schule zurückzudrängen, sondern tritt auch in Konkurrenz zu den Eltern. Solange die Unterhaltung und Errichtung von Schulen weithin als Sache der Kirche angesehen wurde - wobei es den Eltern nach Gutdünken oblag, die Kinder in eine Schule zu schicken oder nicht -, bedurfte es einer Abgrenzung von Erziehungsrechten nicht, da Elternhaus, Kirche und Staat in dem einheitlichen Erziehungsziel, das Kind als gläubigen Christen zu einem gehorsamen Staatsbürger werden zu lassen, kaum in Konflikte grundsätzlicher Art kommen konnten. So bestimmte nach christlicher Moral allein die Kirche die Erziehung des Kindes, und es erübrigte sich daher, Erziehungsrechte voneinander abzugrenzen. ${ }^{1}$ Geprägt durch die klassische katholische Naturrechtslehre konnte danach das staatliche Erziehungsrecht nur subsidiärer Natur sein.

Mit der Durchsetzung der Schulpflicht im 17. Jahrhundert, dem Beginn des Schul-

\footnotetext{
I Erwin Stein, Die rechtsphilosophischen und positiv-rechtlichen Grundlagen des Elternrechts, in: Stein/Joest/Dombois, Elternrecht, Heidelberg 1958, S. 5.
} 
rechts $^{2}$, und der Einführung der Schulaufsicht des Staates im Preußischen Allgemeinen Landrecht und anderen Landrechten treten Elternhaus und Schule jedoch in einen nachhaltigen Interessenkonflikt. Der absolutistische Staat und das Preußische Allgemeine Landrecht, in dessen Tradition sich die gesamte Schulentwicklung in Deutschland vollzog, begründeten mit der Institutionalisierung eines allgemeinen staatlichen Schulwesens den "Primat der staatlichen Schule im Bereich von Bildung und Erziehung « $^{3}$ und erkannten nur noch ein subsidiäres Erziehungsrecht der Eltern an. Mit dem Vorrang der staatlichen Erziehung und der Einführung der Schulpflicht greift der absolutistische Staat grundlegend in das Erziehungsrecht der Eltern besser des Vaters ${ }^{4}$ - ein, wobei von einem Einfluß der Eltern auf die Gestaltung des Schulwesens und von Elternrechten in der Schule nicht die Rede sein kann. ${ }^{5}$ Das Kind ist dabei im naturrechtlichen Verständnis, solange es aufgrund mangelnder eigener Existenzfähigkeit in Elternhaus und Schule lebt, rechtlos und den jeweiligen Erziehungsmitteln und -zielen unterworfen. Dabei scheidet ein Zusammenwirken von Elternhaus und Schule in der Form von Elternrechten in der Schule nicht nur nach dem Selbstverständnis des absolutistischen Staates aus, wonach »der Staat in der Schule über sich selbst, nicht etwa über fremde Rechte verfügt ${ }^{6}{ }^{6}$, sondern auch, weil die objektiven Bedingungen für die Durchsetzung einer allgemeinen öffentlichen Erziehung durch eine bis 1800 fast rein agrarisch geprägte Wirtschaftsweise noch nicht gegeben waren. Die Eltern hatten in ihrer meist bäuerlichen Existenz ${ }^{7}$ kein Interesse an einer schulischen Ausbildung ihrer Kinder, sondern vielmehr an der existenziell notwendigen Mitarbeit, so daß die Einführung der Schulpflicht gleichsam als eine gewaltsame Institutionalisierung öffentlicher Erziehung erscheint, die eine Kooperation von Elternhaus und Schule ausschließ\}t.

Der kindliche Werdegang in der sich entwickelnden bürgerlichen Gesellschaft erfordert die Herstellung einer Persönlichkeit, die in der Lage ist, die Abstraktion bürgerlicher Verkehrsformen zu erfüllen und den Willen und die Fähigkeit besitzt, die individuelle Arbeitskraft zu verkaufen. Dazu aber sind die Familie als alleiniger Erziehungsträger und die Kirche in ihrer traditionellen Wertgebundenheit nicht in der Lage, und so greift der Staat als öffentlicher Erzieher ergänzend in die Sozialisation der Kinder ein. Man kann die Übernahme eines Teils der familiären Erziehung durch die Schule als Ergebnis eines durch Industrialisierung, Technisierung und zunehmende Komplexität bürgerlicher Verkehrsformen bis heute andauernden Prozesses der zunehmenden Vergesellschaftung von Ausbildung und Erziehung begreifen. ${ }^{8}$

Die Aufteilung der Erziehungskompetenzen in Elternrecht und staatliches Schulerziehungsrecht sind jedoch nicht nur als Ausdruck der objektiven Bedingungen der Erziehung von Kindern zu verstehen, sondern darüber hinaus vollzieht sich in der Schule seit der Aufklärung eine Form schulischen Lernens, die für das Kind bis heute nicht nur die Vermittlung des notwendigen Wissens bedeutet, sondern die Verinnerlichung rational-utilitaristischen Denkens, welches als Vernunft erscheint. Geistes-

\footnotetext{
2 Zunächst 1619 im Herzogtum Weimar durch die Weimarsche Schulordnung und danach sukzessive in allen deutschen Staaten, so in Preußen durch die principia regulativa 1717

3 Hans-Uwe Erichsen, Verstaatlichung der Kindeswohlentscheidung, Berlin, New York 1979, S. I4.

4 Das Elternrecht bestand weitgehend in Form der im Hause unangetasteten spatria potestas<.

5 Theo Bingler, Studien zum Elternrecht bei den Naturrechtsphilosophen des 16. und 17. Jahrhunderts, Diss. Freiburg 1960, S. 99.

6 Günter Frankenberg, Verrechtlichung schulischer Bildung, Diss. München I 978, S. I5.

7 Aber auch aufgrund ihres geringen Lohnes als Lohnarbeiter war das Interesse der Eltern an einer schulischen Ausbildung ihrer Kinder gering.

8 Peter Büchner (Hrsg.): Die Eltern und die Schule, München 1976, S. 29.
} 
geschichtlich wird dementsprechend das Zeitalter der Aufklärung zum Zeitalter der

Pädagogik. ${ }^{9}$

Dabei ist entscheidend, daß in der Schule zwar auch Fähigkeiten von Lesen, Rechnen und Schreiben vermittelt werden, der Unterricht jedoch nicht primär auf kognitive Wissensvermittlung, sondern auf Disziplinierung und Untertanengeist abgestellt ist, ${ }^{10}$ und zwar im Sinne auch einer Selbstdisziplinierung, die das Individuum dazu disponiert, von sich und seinen Bedürfnissen zu abstrahieren. Lust, Sinnlichkeit und Gefühl müssen einer Vernunft weichen, die nur zuläßt, was nützlich ist, wobei "Arbeit« zum zentralen anthropologischen Begriff wird. " Die Abstraktion als entscheidendes Werkzeug aufklärerischen Denken $s^{12}$ und schulischen Lernens ist im Gegensatz zur »reinen Erfahrenswelt « in der Familie bestimmt vom Moment der Trennung von Subjekt und Objekt, Sinnlichkeit und Verstand, der Gleichsetzung von Mathematik und Denken. Dabei soll »das moderne Kind lernen, seine mimetischen Impulse zu zügeln und auf ein bestimmtes Ziel zu lenken ${ }^{13}$ Dies soll die Vernunft leisten, die nach Kant der Mensch als höchstes Erziehungsziel zur Beherrschung von Leidenschaften und spontanen Intentionen zu verinnerlichen hat. Ein Mittel dazu ist die Disziplinierung des Kindes. "Disziplin unterwirft den Menschen den Gesetzen der Menschheit und fängt an, ihn den Zwang der Gesetze fühlen zu lassen. Dieses muß aber frühe geschehen. So schickt man z. B. Kinder anfangs in die Schule, nicht schon in der Absicht, damit sie dort etwas lernen sollen, sondern damit sie sich daran gewöhnen, stillzusitzen und pünktlich das zu beobachten, was ihnen vorgeschrieben wird, damit sie nicht in Zukunft jeden ihrer Einfälle wirklich auch augenblicklich in Ausübung bringen mögen. «" ${ }^{13 a}$ Wenn Disziplinierung dabei für Kant die Bezähmung der wilden Freiheit zur Erreichung der sittlichen Freiheit als praktische Vernunftautonomie darstellt, so wiederholt sich in der daraus resultierenden Selbstdisziplinierung des Subjekts, unter das, was die Vernunft gebietet, die Unterwerfung der Natur durch den Menschen im Kinde, wobei die Vernunft nicht mehr positiv dazu dient, die Affekte zu beherrschen, sondern zu unterdrücken.

In der Weimarer Reichsverfassung (WRV) fand die Bedeutung der allgemeinen öffentlichen Erziehung in den Schulartikeln ihren Ausdruck, denen das Elternrecht lediglich deklamatorisch gegenüberstand. Auch wenn in Art. I 20 WRV die Erziehung des Nachwuchses zur leiblichen, seelischen und gesellschaftlichen Tüchtigkeit als die oberste Pflicht und das natürliche Recht der Eltern, worüber die staatliche Gemeinschaft wacht, bezeichnet wurde, konstituierte sich das Verhältnis von elterlichem und schulischem Erziehungsrecht nach dem Prinzip des Vorzugs der öffentlichen Erziehung vor der privaten im Sinne der preußisch-deutschen Überlieferung. Während Art. I20 WRV lediglich als Einrichtungsgarantie oder Programmsatz ${ }^{14}$ angesehen wurde, gehörten die Schulartikel 142 bis 149 WRV zu den wichtigsten Grundsätzen des Zusammenlebens der staatlichen Gemeinschaft. "s

9 Bodo Voigt, Bildungspolitik und politische Erziehung in den Klassenkämpfen, Frankfurt I973, S. 66. to Vgl. z. B. den Lernzielkatalog für Preußen 1829 bei Klaus Schmitz, Geschichte der Schule, Stuttgart 1980 , S. 76. Soweit heute das Erziehungsziel Mündigkeit< proklamiert wird, zeigt dies, inwieweit an die Stelle der notwendigen Erziehung zum Untertanen im Übergang von feudaler zu bürgerlicher Gesellschaft mit der Durchsetzung bürgerlicher Verkehrsformen die Fähigkeit der Selbstdisziplinierung des Kindes getreten ist.

I I Voigt, a. a. O., S. 99.

I2 Max Horkheimer, Theodor W. Adorno, Dialektik der Aufklärung, Frankfurt 1969, S. I s.

13 Max Horkheimer, Zur Kritik der instrumentellen Vernunft, Frankfurt 1974, S. I I 3 .

I 3 a Immanuel Kant, Vorlesungen über Pädagogik, in: Kants Werke, Akademie Ausgabe, Berlin I968, Bd. IX, S. 90.

I4 Vgl. Gerhard Anschütz, Die Verfassung des Deutschen Reichs vom 11. August 1919, 14. Aufl. 1933 (Nachdruck), Bad Homburg v. d. H., 1968, Art. I 20 Anm. 3.

is Vgl. Hans Peters, Elternrecht, Erziehung, Bildung und Schule in: Bettermann, Nipperdey, Scheuner, Die Grundrechte, Berlin, 1960, Bd. IV, S. 37 r. 
Neben dem Prinzip "Staatsrecht überhöht Elternrecht" galt unter der WRV das Separationsprinzip von elterlicher und schulischer Erziehung, d. h. einer strikten Trennung von familiären und schulischen Kompetenzen. Geprägt von der Vorstellung einer grundsätzlich unbeschränkten staatlichen Schulhoheit oblag dem Staat danach die uneingeschränkte Organisationsgewalt und das Bestimmungsrecht in der Schule. ${ }^{16}$ Wurden Rechte der Eltern in der Schule auf diese Weise zurückgewiesen, so war auch unter der Geltung der WRV zwischen Eltern und Schule unzweifelhaft, daß ihnen nach eigenem Ermessen die Erziehungsmittel zustanden und das Kind insoweit keine Rechte hatte. Unter dem Einfluß der Reformpädagogik entwickelten sich dennoch Kooperationsformen zwischen Elternhaus und Schule, die gleichwohl das Primat der staatlichen Erziehung nicht erschütterten.

Die Nationalsozialisten schließlich benutzten die öffentlichen Schulen zur Indoktrination der Kinder mit faschistischem Gedankengut, Elternmitarbeit in der Schule, vor allem in der Form von Elternbeiräten als Ausdruck einer emanzipatorischdemokratischen Forderung nach mehr Einfluß der Eltern auf die Schule, wurde unterbunden; stattdessen unterstanden alle Eltern dem Schulleiter und bildeten die "Schulgemeinde ${ }^{17}{ }^{7}$, wobei jeglicher Einfluß der Eltern auf die Gestaltung der Erziehung der Jugend im allgemeinen und der Schule im besonderen fortschreitend zersetzt wurde.

\section{Elternrecht und staatliches Schulerziehungsrecht unter der Geltung des GG}

Während das Erziehungsrecht der Eltern in der WRV durch seine Normierung in Art. I20 WRV Berücksichtigung findet, ohne sich dabei vom Primat der staatlichen Erziehung zu befreien, beendet das Grundgesetz die Subsidiarität des Erziehungsrechts der Eltern gegenüber dem staatlichen Schulerziehungsrecht. Nach Art. 6 Abs. 2 GG sind Pflege und Erziehung der Kinder das natürliche Recht und die zuvörderst den Eltern obliegende Pflicht, welches allgemein unter der Geltung des GG als Grundrecht im klassischen Sinne anerkannt ist. ${ }^{18}$ Neben dem elterlichen Erziehungsrecht steht das Schulerziehungsrecht des Staates, welches zunächst positiv im GG nicht ausdrücklich normiert ist, sondern sich aus der Schulaufsicht des Staates gem. Art. 7 Abs. I GG ergibt. Zur Begründung wird entweder darauf verwiesen, daß Art. 7 Abs. I GG ein staatliches Schulerziehungsrecht voraussetze ${ }^{19}$, oder daß unter dem staatlichen Schulauftrag auch immer ein Erziehungsauftrag zu verstehen sei. ${ }^{20}$ Unter ausdrücklicher Berufung auf die historische Entwicklung der staatlichen Schulaufsicht ${ }^{21}$ wird damit der seit der Institutionalisierung allgemeiner öffentlicher Schulen erhobene Anspruch des Staates, "Erzieher der Kinder « zu sein, auch in das Grundgesetz aufgenommen. ${ }^{22}$

I6 Vgl. Walter Landé, Die staatsrechtlichen Grundlagen des deutschen Unterrichtswesens, in: Handbuch des Deutschen Staatsrechts, hrsg. v. G. Anschütz, R. Thoma, Tübingen 1932, II. Band, S. $703 \mathrm{ff}$. (720).

17 Manuela de Bois-Reymond, Verkehrsformen zwischen Elternhaus und Schule, Frankfurt 1977, S. 98.

18 Vgl. z. B. F. Klein, in: H. v. Mangoldt/F. Klein, Das Bonner Grundgesetz, 2. Aufl., Berlin u. Frankfurt 1957, Bd. I Art. 6 Anm. IV, 2; Th. Maunz, in: Th. Maunz, G. Dürig, R. Herzog, R. Scholz, Grundgesetz Kommentar, Stand Sept. 1980, Art. 6 Rdnr. 6; E. M. v. Münch, in: I. v. Münch (Hrsg.), Grundgesetz Kommentar, Bd. I Frankfurt 1974, Art. 6 Rdnr. I5; BVerfGE 4, 52 (57); 7, 320 (323); 24, I19 (138).

I9 BVerfGE $34,165(183) ; 4$ I, $29(44) ; 47,46(72)$.

20 Ursula Fehnemann, Bemerkungen zum Elternrecht in der Schule, DÖV 1978, 489 (491) m. w. N

21 BVerwGE 6, 101 (104); 18, $3^{8}$ (39), 40 (4I); 21,289 (290); 47, 194 (198); BVerfGE 47, 46 (80).

22 Aber noch heute vertreten z. B. Ossenbühl (Schule im Rechtsstaat, DÖV 1980, 801 (808)) und Erichsen 
Die Normierung des Elternrechts in Art. 6 Abs. 2 GG und des staatlichen Schulerziehungsrechts in Art. 7 Abs. I GG sind Ausdruck einer bewußten Entscheidung des Parlamentarischen Rates. Einerseits sollte durch die Normierung in zwei aufeinanderfolgenden Artikeln des Grundgesetzes der enge Zusammenhang von Elternrecht und staatlichem Schulerziehungsrecht bei gleichzeitiger Grundrechtsanerkennung beider Erziehungsrechte dokumentiert werden; andererseits wurde mit der Fassung der Art. 6 und 7 GG der Antrag von CDU und Zentrumspartei zurückgewiesen, die Regelungen über das Elternrecht und das Schulwesen in einem Artikel des Grundgesetzes zu fassen. ${ }^{23}$ Trotz der Ablehnung eines klassischen konfessionellen Elternrechts in der Schule wurde der konfessionelle Einfluß der Eltern dadurch gewährleistet, daß die Eltern als »echtes Grundrecht« gem. Art. 7 Abs. 2 GG über die Teilnahme des Kindes am Religionsunterricht bestimmen können ${ }^{24}$ und daß gem. Art. 7 Abs. s GG die Möglichkeit besteht, private Volksschulen als Bekenntnisschulen einzurichten.

Elternrecht und staatliches Schulerziehungsrecht stehen als eigenständige, unabgeleitete Rechte nebeneinander. Nach der Rechtsprechung des Bundesverfassungsgerichts ist der allgemeine Auftrag der Schule zur Bildung und Erziehung der Kinder dem Elternrecht in der Schule nicht nach-, sondern gleichgeordnet ${ }^{25}$, so daß weder dem Elternrecht noch dem Erziehungsauftrag des Staates ein absoluter Vorrang zukommt und der Staat daher unabhängig von den Eltern eigene Erziehungsziele verfolgen kann. ${ }^{26}$ Dabei ist die "gemeinsame Erziehungsaufgabe von Eltern und Schule, welche die Bildung der einen Persönlichkeit des Kindes zum Ziel hat . . . in einem sinnvoll aufeinander bezogenen Zusammenwirken zu erfüllen «. ${ }^{27}$ Dieses begründet auch Rechte der Eltern in der Schule.

Denn »aus Art. 7 Abs. 2 und , GG ... kann nicht geschlossen werden, daß dieser Artikel des Grundgesetzes als lex specialis allein und abschließend die Reichweite des Elternrechts im Schulwesen regele ... Gegenüber der Vorstellung einer grundsätzlich unbeschränkten staatlichen Schulhoheit, wie sie die Weimarer Reichsverfassung beherrschte..., hat das Grundgesetz innerhalb des Gesamtbereichs >Erziehunge das individualrechtliche Moment verstärkt und den Eltern, auch soweit sich die Erziehung in der Schule vollzieht, größeren Einfluß eingeräumt, der sich in Art. 6 Abs. 2 S. I GG zu einer grundrechtlich gesicherten Position verdichtet hat ${ }^{28}{ }^{28}$

Diese von der Rechtsprechung entwickelten Ausprägungen des Elternrechts in der Schule lassen sich verschiedenen Bereichen zuordnen. So wird den Eltern aus Art. 6 Abs. 2 GG ein Anspruch auf die organisatorische Gestaltung des Scbulwesens eingeräumt. Danach hat der Staat in der Schule die Verantwortung der Eltern für den Gesamtplan der Erzichung ihrer Kinder zu achten und darf daher durch schulorganisatorische Maßnahmen nie den ganzen Werdegang des Kindes regeln wollen. ${ }^{29}$ Aus diesem Grund obliegt den Eltern nach dem Besuch der allgemein verpflichtenden Grundschule zunächst prinzipiell die Wahl zwischen den möglichen weiterführenden Schulformen. ${ }^{30}$ Darüber hinaus ist es dem Staat verwehrt, die Kinder übermäßig

a. a. O., die Position, der Schule obliege in erster Linie reine Wissensvermittlung und sie dürfe nur als notwendiger Annex zur Wissensvermittlung erzieherisch tätig werden, womit sie als Mindermeinung die Funktion von Schule völlig verkennen.

23 Vgl. die Entwicklungsgeschichte von Art. 6 und 7 GG; JböffR NF I (1951), S. 102 ff.

24 Begrenzt durch die Religionsmündigkeit des Kindes mit 14 Jahren gem. $\$$ s RelErzG.

25 BVerfGE 34, I6s (I 83 ); 41, 29 (44); 47, 46 (72); ebenso BVerwGE 5, I53 (I55).

26 BVerfGE 47 , a. a. O.

27 BVerfGE 34, 16s (182); 47, 46 (74)

28 Ebd.

29 BVerfGE 34,165 ( $\left.18_{3}\right)$.

30 A. a. O., S. I 44 f.; BVerwGE 5, 153 (I56 f.) 
lange in einem "undifferenzierten Unterricht festzuhalten « $^{31}$, welches einen Anspruch der Eltern auf eine differenzierte Organisation des Schulwesens in verschiedene Schularten impliziert. So liegt »die Grenze des verfassungsrechtlich Zulässigen dort ..., wo das Wahl- und Bestimmungsrecht der Eltern angesichts nur noch einer einzigen vorhandenen obligatorischen Schulform mit einem vom Staat einseitig festgelegten Bildungsziel obsolet wird und leerläuft «.$^{32}$

In Abgrenzung ihres Einflusses auf die organisatorische Gestaltung des Schulwesens können die Eltern aus Art. 6 Abs. 2 GG jedoch nicht das Recht herleiten, daß der Staat eine ihnen wunschgemäße Schulform zur Verfügung stellt, ${ }^{33}$ was insbesondere bedeutet, daß »ein positives Bestimmungsrecht, aufgrund dessen die Eltern vom Staat die Einrichtung von Schulen bestimmter religiöser oder weltanschaulicher Prägung verlangen könnten «, nicht besteht. ${ }^{34}$ Organisatorische Gliederung der Schule, strukturelle Festlegung des Ausbildungssystems, inhaltliches und didaktisches Konzept und die Bestimmung der Lernziele sowie die Entscheidung darüber, ob und inwieweit diese Ziele von dem Schüler erreicht worden sind, sind zunächst grundsätzlich dem Bestimmungsrecht der Eltern entzogen und gehören zum staatlichen Gestaltungsbereich. ${ }^{35}$

Trotz dieser grundsätzlichen Bestimmungsgewalt des Staates in der Schule wird den Eltern ein Einfluß auf die inhaltliche Ausgestaltung des Schulwesens eingeräumt. Dieser wird wesentlich durch das Toleranzgebot umrissen. Als ein nach Ansicht Oppermanns nötigenfalls einklagbares "Grundrecht der Schüler und Eltern auf eine ideologisch tolerante Schule ${ }^{36}$ soll es als Kernpunkt das Verbot der Indoktrination und einseitiger ideologischer Beeinflussung der Kinder im Rahmen des Unterrichts umfassen. ${ }^{37}$ Hinsichtlich der negativen und positiven Religionsfreiheit der Eltern ist dieses Spannungsverhältnis entsprechend dem "grundgesetzlichen Gebor der Toleranz« dahingehend zu lösen, daß sich mit der Einführung christlicher Bezüge im Schulwesen nur ein Minimum von Zwangselementen bezüglich der Glaubens- und Gewissensfreiheit verbinden darf und diese nicht christlich-konfessionell fixiert sein dürfen. ${ }^{38}$ Desweiteren konkretisiert das Bundesverfassungsgericht in der Sexualkunde-Entscheidung Ansprüche der Eltern auf die inhaltliche Gestaltung des Schulwesens dahingehend, daß die Eltern bei der Durchführung der schulischen Sexualerziehung die gebotene Zurückhaltung und Toleranz verlangen können und die Schule jeden Versuch der Indoktrination der Schüler mit dem Ziel, ein bestimmtes Sexualverhalten zu befürworten oder abzulehnen, zu unterlassen habe. ${ }^{39}$

Aus Art. 6 Abs. 2 GG ergeben sich weiterhin bestimmte Verfahrens- und Informationsrechte der Eltern in der Schule. So unterliegen die wesentlichen schulorganisatorischen Maßnahmen, die das Elternrecht betreffen, nach dem Abschied vom »besonderen Gewaltverhältnis « dem Gesetzesvorbehalt, wobei unter »wesentlich" bezogen auf den grundrechtsrelevanten Bereich »wesentlich für die Verwirklichung

31 BVerfGE 34, 165 (184).

32 BVerfGE 45, 400 (416).

33 BVerfGE 34, I65 (185); 45, 400 (415).

34 BVerfGE 4I, 29 (46); es besteht auch kein Recht auf die Einführung des Schulgebets, jedoch in sehr zweifelhafter Abgrenzung die Befugnis, dies auf freiwilliger Basis in den Schultagesablauf einzubauen; vgl. BVerfG DÖV 1980, 333 ff.

35 BVerfGE 34, I65 (182); 45, 400 (41 5); gleichwohl bleibt z. B. offen, ob nicht etwa die Ganztagsschule als Regelschule gegen das Elternrecht aus Art. 6 Abs. 2 GG verstößt.

36 Thomas Oppermann, Nach welchen rechtlichen Grundsätzen sind das öffentliche Schulwesen und die Stellung der an ihm Beteiligten zu ordnen? Gutachten C für den 51. DJT, München 1976, S. 94.

37 Thomas Oppermann, Zum Grundrecht auf eine tolerante Schule, RdJB 1977, S. 44 f.

38 BVerfGE $4 \mathrm{I}, \mathrm{si} \mathrm{f}$.

39 BVerfGE $47,46(77)$. 
der Grundrechte « zu verstehen ist. ${ }^{\circ}$ »Auch im Schulverhältnis spielt die Grundrechtsrelevanz eine erhebliche Rolle. Die Grenze zwischen dem staatlichen Erziehungsauftrag (. . .) und dem Elternrecht (. . .) sowie den Persönlichkeitsrechten des Kindes (...) sind oft flüssig und nur schwer auszumachen. Ihre Markierung ist für die Ausübung dieser Grundrechte vielfach von maßgebender Bedeutung. Sie ist daher Aufgabe des Gesetzgebers «. ${ }^{41}$

Außerdem steht den Eltern ein individuelles Informationsrecht über Vorgänge in der Schule zu, »deren Verschweigen die Ausübung des individuellen elterlichen Erziehungsrechts beeinträchtigen könnte " ${ }^{42}$ So haben die Eltern ein Recht auf Information über die schulischen Leistungen ihres Kindes ${ }^{43}$ und über beabsichtigte Ein- und Umstufungen ${ }^{44}$, über Inhalt und methodisch-didaktischen Weg der Sexualerziehung ${ }^{45}$, über die beabsichtigte Veranstaltung eines Schulgebets und die Möglichkeit der Nichtteilnahme ${ }^{46}$ und ähnliche Vorgänge in der Schule. Dieses Informationsrecht findet dort seine Grenze, wo »in besonders gelagerten Fällen eine Information der Eltern zu Reaktionen führen kann, die im Interesse des Kindeswohls nicht zu verantworten sind $\ll .47$

Neben diesen individuellen Rechten aus Art. 6 Abs. 2 GG stehen den Eltern kollektive Mitwirkungsrechte in der Schule zu, die in den Schulgesetzen der Länder festgelegt sind. Es handelt sich dabei im wesentlichen um Anhörungs und Informationsrechte in der Form von Elternvertretungen auf den verschiedenen Ebenen..$^{8}$ Diese Mitwirkungsrechte der Eltern in der Schulverwaltung können nach der Rechtsprechung des Bundesverfassungsgerichts nicht aus Art. 6 Abs. 2 GG hergeleitet werden ${ }^{49}$, sondern sind im Rahmen des Rechtsstaats-, Demokratie- und Sozialstaatsgebots Sache der Länder bei der Ausgestaltung des Schulwesens gem. Art. 7 Abs. I GG.

So unstreitig die Notwendigkeit einer Kooperation von Elternhaus und Schule sein dürfte, weil die Erkenntnisse der Sozialisationsforschung zeigen, daß sich schulische Lernprozesse sehr wesentlich auf Potentiale stützen, die durch den familiären Sozialisationsprozeß geschaffen werden und der Lernerfolg in der Schule zu einem nicht unwesentlichen Teil vom familiären Kontext abhängt ${ }^{50}$, eine Kooperation daher notwendig ist, um die kindliche Entwicklung nicht durch unverarbeitete gegensätzliche Wert-, Verhaltens- und Sprachebenen zu behindern ${ }^{\text {SI }}$, so bleibt dennoch die Frage, ob nicht durch die Begründung von Rechten der Eltern in der Schule »die spezifischen gesellschaftlichen Funktionsbereiche des Art. 6 und 7 GG ... verschwimmen ".2 Indem das Bundesverfassungsgericht den Eltern aus Art. 6 Abs. 2 GG bestimmte individuelle Rechte in der Schule einräumt, ist anerkannt, daß Art. 6

40 A. a. O., S. 79, so z. B. die Festlegung der Erziehungsziele und Unterrichtsprinzipien bei der Sexualerziehung.

4 I A. a. O., S. 80.

42 BVerfG DVBI 1982, 406 (407)

43 Vgl. Fritz Ossenbühl, Erziehung und Bildung, AöR 98, 1973, 361 (367) mit Rechtsprechungshinweisen der Oberverwaltungsgerichte.

44 BVerfGE 34, 165 (192).

45 BVerfGE $47,46(76)$.

46 BVerfG DÖV I $980,333(336)$

47 BVerfG DVBI I 982,406 (408)

48 Über die prinzipielle Mitwirkungsbefugnis besteht weitgehend Einigkeit, für die zukünftige Entwicklung vgl. den Entwurf eines Landesschulgesetzes der Kommission des DJT, RdJB 1981, S. 224 ff.

49 BVerfG DVBI I982, 406 (407).

so Büchner, a. a. O. (Fn. 8), S. I 2 .

s I Barbara Rothgang, Gerhard Wurzbacher, Die Übernahme familialer Erziehungsaufgaben und -ansprüche durch gesellschaftliche Institutionen, in: Klaus Schleicher (Hrsg.), Elternmitsprache und Eiternbildung, Düsseldorf 1973, S. 21 .

S2 U. K. Preuß, Lehrplan und Toleranzgebot, RdJB 1976, 267 (268). 
Abs. 2 GG nicht nur als Abwehrrecht gegen unzulässige staatliche Eingriffe, sondern als individuelles Teilhaberecht zu verstehen ist. Einem Verständnis des Elternrechts aus Art. 6 Abs. 2 GG als Teilhaberecht ist prinzipiell zuzustimmen.

Gegenwärtig ist es nicht ungefährlich, die Schule so dem Hegemonieanspruch der Mittelschicht zu unterwerfen, da individuelle Rechte und Mitwirkungsrechte der Eltern in schulischen Gremien wesentlich mittelschichtsorientiert sind. ${ }^{3}$ So sind die Auseinandersetzungen um die Förderstufe, Sexualerziehung, integrierte Gesamtschule, Oberstufenreform und Rahmenrichtlinien der Versuch, über das Elternrecht gesellschaftspolitisch mißliebige Bewegungen einzudämmen. ${ }^{54}$ Deutlich wird diese tagespolitische Instrumentalisierung des Elternrechts auch bei der Frage um das Toleranzgebot in der Schule. Bezeichnend ist dabei, daß das Gebot der Toleranz in der juristischen Diskussion in dem Augenblick ausdrücklich geltend gemacht wurde, als es darum ging, politisch mißliebige Bildungsreformen zurückzudrängen. Die Forderung nach einer "toleranten Schule erheben dabei jene am lautesten, denen es um Toleranz im klassischen Sinne gar nicht geht. Indem die Schule, gestützt auf eine demokratische Bildungsbewegung, emanzipatorische Prozesse einzuleiten versucht, wird die Forderung nach Toleranz unter Berufung auf das Elternrecht zum wesentlichen Argument konservativer Eltern. Das macht gegenwärtig die Berufung auf das einklagbare Grundrecht der Eltern aus Art. 6 Abs. 2 GG auf eine tolerante Schule problematisch. Zudem muß die Unterrichtsgestaltung nach Maßgabe der Bildungsund Erziehungsziele der Länderverfassungen und der Schulgesetze der Länder aufgrund des eigenständigen Erziehungsauftrages des Staates in der Schule gem. Art. 7 Abs. I GG erfolgen. Das Elternrecht gewährt zwar einen Anspruch auf Teilhabe und wird daher wesentlich geprägt durch Informations-, Anhörungs- und organisatorische Beteiligungsrechte, kann sich jedoch nicht auf die inhaltliche Ausgestaltung des Unterrichts ausweiten, da ansonsten das staatliche Erziehungsrecht in unzulässiger Weise eingeschränkt wird. Der prinzipiellen Begründung von Rechten der Eltern in der Schule ist jedoch zuzustimmen, da »ein fortgeschrittenes Demokratieverständnis, das auf die Teilung von Herrschaft und auf die Durchsetzung individueller und kollektiver Interessen abzielt, (. . .) sich nur dort verwirklichen (kann), wo die Menschen ihre unmittelbaren Interessen haben. Dazu gehören nicht primär die Parlamente, sondern gesellschaftliche Bereiche wie Schule und Arbeitsstättew." Der Staat sollte aus diesem Grund im Rahmen seiner Schulhoheit die Eltern auch an der Gestaltung des Unterrichts beteiligen, ein Anspruch darauf läßt sich aus Art. 6 Abs. 2 GG jedoch nicht herleiten.

\section{Die freie Entfaltung der Persönlichkeit des Kindes}

Rechte der Eltern in der Schule gewährleisten gleichwohl noch nicht, was im Zentrum der Verrechtlichung schulischer Beziehungen stehen sollte: die Voraussetzung für die freie Entfaltung der Persönlichkeit des Kindes zu schaffen. Es ist vielmehr notwendig zu untersuchen, inwieweit Rechte der Eltern in der Schule in Abgrenzung zum staatlichen Schulerziehungsrecht mit den Grundrechten des Kindes korrespondieren.

Das Bundesverfassungsgericht begründet das von den Eltern wahrzunehmende

53 Vgl. de Bois-Reymond, a. a. O. (Fn. 17), S. 118.

54 Vgl. dazu Ingo Richter, Elternrecht, in: ders. (Hrsg.), Bildungsverfassungsrecht, Berlin 1973, S. 61.

is Wolfgang Mickel, Das Elternrecht im Schulwesen der Bundesrepublik, RdJB 1974, 363 (364). 
Recht auf freie Wahl zwischen den verschiedenen zur Verfügung gestellten Schularten damit, daß »der Staat durch schulorganisatorische Maßnahmen nie den ganzen Werdegang des Kindes regeln wollen (darf).$^{56}$ Damit werde berücksichtigt, "daß sich das Leben des Kindes nicht nur nach seiner ohnehin von Umweltfaktoren geprägten Bildungsfähigkeit und seinen Leistungsmöglichkeiten gestaltet, sondern daß hierfür auch die Interessen und Sozialvorstellungen der Familie von großer Bedeutung sind. Diese primäre Entscheidungszuständigkeit der Eltern beruht auf der Erwägung, daß die Interessen des Kindes am besten von den Eltern wahrgenommen werden 4.57

Danach soll den Eltern der Gesamterziehungsplan für die Erziehung ihrer Kinder obliegen, da sie als die natürlichen Sachverwalter für die Erziehung ihrer Kinder die Interessen der Kinder am besten wahrnehmen. ${ }^{8}{ }^{8}$ Indem das Kind in das gesellschaftliche Leben und seine Zusammenhänge hereinwächst, kann man jedoch nicht mehr einfach als "Norm der Natur" davon ausgehen, „daß die Eltern sich gleichsam instinktiv vom Kindeswohl leiten lassen ${ }^{59}$ oder wie das Bundesverfassungsgericht a priori annehmen, daß die Eltern als die natürlichen Sachverwalter für die Erziehung ihrer Kinder die Interessen des Kindes am besten wahrnehmen. ${ }^{\circ} \mathrm{Da}$ jede Erziehung eine gesellschaftlich bedingte ist, gibt es keine menschliche Natur außerhalb oder vor der Gesellschaft, aus der ein Erziehungsrecht der Eltern rational-konstruktiv oder vernunftgemäß abgeleitet werden könnte. So wie es keine ewige Menschennatur gibt und »kein fixes Gattungswesen Mensch mit statischen Eigenschaften, auf die ein Naturrecht zu gründen wäre«, sondern die gesamte Geschichte vielmehr eine fortgesetzte Umwandlung der menschlichen Natur darstellt, so wenig gibt es eine menschliche "unwandelbare und normative Gesamtnatur " ${ }^{61}$, die außerhalb der Gesellschaft steht. Es besteht vielmehr ein dialektisches Verhältnis von Natur und Gesellschaft, in welches auch das Verhältnis der Eltern zu ihren Kindern eingebettet ist. Die Ablehnung einer naturrechtlichen Konstruktion des Elternrechts und die Kritik der sogenannten natürlichen Voraussetzungen der Erziehung schließt dabei keinesfalls eine Abwehr des Natürlichen ein. Sie besagt vielmehr, daß diese nur als historisch-gesellschaftlich beeinflußte natürliche Voraussetzungen verstanden werden können. ${ }^{62}$ Man muß nicht wie Heinsohn/Knieper ${ }^{63}$ von einem objektiven Widerspruch der Interessen von Lohnarbeitern an der »Aufzucht « von Kindern ausgehen, um angesichts gesellschaftlicher Erscheinungen wie Kindesvernachlässigung und -mißhandlung die Annahme, die Eltern würden natürlicherweise die Interessen des Kindes am besten wahrnehmen, zu bezweifeln. Des Dilemmas zwischen Ideal und Realität ist sich das Bundesverfassungsgericht bewußt, wenn es zur allein den Eltern obliegenden Entscheidung über die Schullaufbahn des Kindes ausführt, daß »sogar die Möglichkeit in Kauf genommen wird, daß das Kind durch einen Entschluß der Eltern Nachteile erleidet, die im Rahmen einer nach objektiven Maßstäben betriebenen Begabtenauslese vielleicht vermieden werden könnten ${ }^{64}$

Es bleibt der der Unmündigkeit und dem Objektdenken gegenüber dem Kind verhafteten Position nur die Lösung, die Entscheidungskompetenz über die Schullauf-

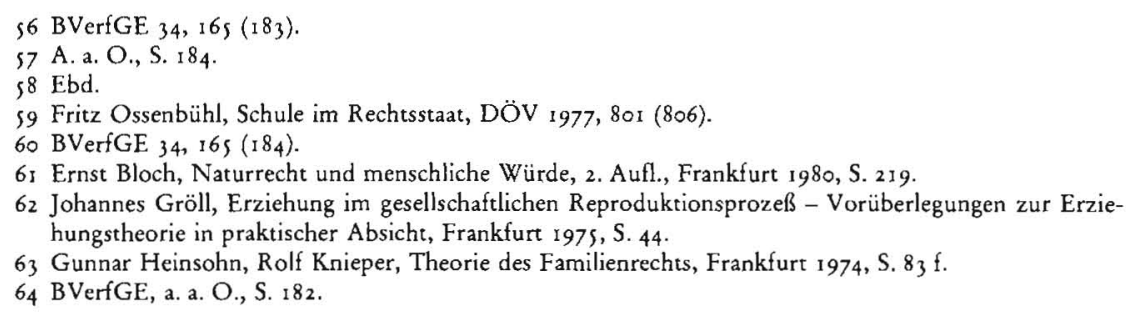


bahn des Kindes entweder den Eltern oder der Schule zu überlassen. Vom Recht des Kindes her auf freie Entfaltung seiner Persönlichkeit und der Freiheit der Berufswahl gäbe es jedoch gerade jenseits der selektiven Artenvielfalt des Schulwesens die Möglichkeit, ein Schul- und Bildungssystem zu gewährleisten, bei dem bis zur Entwicklung des Kindes zu eigener Entscheidungsfähigkeit von Eltern oder Schule keine Entscheidung getroffen werden können, die für das Kind nachteilig sind. Dies würde bedeuten, gerade entgegen den vom Bundesverfassungsgericht entwickelten Kriterien eine möglichst lange Schulausbildung in einer Schulart zu gewährleisten, um dem Kind bei genügender Reife die Entscheidungskompetenz über die weitere Schullaufbahn selbst zu belassen. Da dies eine Differenzierung nicht ausschließt, könnte auch insofern den verschiedenen Begabtenrichtungen Raum gelassen werden, ohne jedoch das Kind durch Entscheidungen eines selektiven Schulsystems zu benachteiligen. So würde einerseits eine Kompetenzzuweisung zwischen Elternhaus und Schule nicht über das Objekt "Kind « hinweg stattfinden; andererseits müßten quasi absolut gesetzte Prämissen im Verhältnis von Elternrecht und staatlichem Schulerziehungsrecht neu überdacht werden. Damit wäre auch die Abgrenzung von Elternrecht und staatlichem Schulerziehungsrecht seiner teilweisen Beliebigkeit entzogen und eine Verobjektivierung von Entscheidungen zum Wohle des Kindes möglich, die die generalklauselartige Formulierung eines sinnvollen Zusammenwirkens der Erziehungsträger unter Einbeziehung des Kindes mit Inhalt füllt. Hierbei muß sich das Kind mittels seiner Grundrechtsmündigkeit ${ }^{6 s}$ auf das Recht der freien Entfaltung der Persönlichkeit berufen können, soweit Eltern- und Kindesinteressen in diesem Bereich zuwiderlaufen. Es erscheint dies als die rechtliche Konsequenz der gesellschaftlichen Entwicklung der bürgerlichen Familie.

Frank-Rüdiger Jach

6s Auf das besondere Problem der unzulässigen Trennung von Grundrechtsfähigkeit, -mündigkeit und der Drittwirkung von Grundrechten im Eltern-Kind-Verhältnis soll hier nicht weiter eingegangen werden. 